\title{
Stellar Populations of Kinematically Decoupled Cores in E/S0 Galaxies $\dagger$
}

\author{
Richard M. McDermid ${ }^{1}$, Eric Emsellem ${ }^{2}$, Kristen L. Shapiro ${ }^{3}$, \\ Roland Bacon $^{2}$, Martin Bureau ${ }^{4}$, Michele Cappellari ${ }^{1,4}$, Roger L. \\ Davies $^{4}$, Tim de Zeeuw ${ }^{1}$, Jesús Falcón-Barroso ${ }^{1,5}$, Davor Krajnović ${ }^{4}$, \\ Harald Kuntschner ${ }^{6}$ Reynier F. Peletier ${ }^{7}$, and Marc Sarzi ${ }^{8}$ \\ ${ }^{1}$ Leiden Observatory, Leiden University, Postbus 9513, 2300 RA Leiden, The Netherlands \\ email: mcdermid@strw.leidenuniv.nl \\ ${ }^{2}$ Université de Lyon 1, CRAL, Observatoire de Lyon, 9 av. Charles André, F-69230 \\ Saint-Genis Laval; CNRS, UMR 5574 ; ENS de Lyon, France \\ ${ }^{3}$ UC Berkeley Department of Astronomy, Berkeley, CA 94720, USA \\ ${ }^{4}$ Denys Wilkinson Building, University of Oxford, Keble Road, Oxford, United Kingdom \\ ${ }^{5}$ European Space and Technology Centre (ESTEC), Keplerlaan 1, Postbus 299, 2200 AG \\ Noordwijk, The Netherlands \\ ${ }^{6}$ Space Telescope European Coordinating Facility, European Southern Observatory, \\ Karl-Schwarzschild-Str 2, 85748 Garching, Germany \\ ${ }^{7}$ Kapteyn Astronomical Institute, Postbus 800, 9700 AV Groningen, The Netherlands \\ ${ }^{8}$ Centre for Astrophysics Research, Science \& Technology Research Institute, University of \\ Hertfordshire, Hatfield, United Kingdom
}

\begin{abstract}
In this poster contribution, we present results from high spatial resolution integralfield spectroscopy of elliptical (E) and lenticular (S0) galaxies from the SAURON representative survey, obtained with the OASIS and GMOS spectrographs. These seeing-limited observations explore the central $\sim 10^{\prime \prime} \times 10^{\prime \prime}$ (typically one kiloparsec diameter) regions of these galaxies using a spatial sampling four times higher than SAURON (0.' 27 vs. 0.94 spatial elements), resulting in almost a factor of two improvement in the median PSF. These data allow accurate study of the central regions to complement the large-scale view provided by SAURON. We derive the stellar and gas kinematics, stellar absorption-line strengths and nebular emission-line strengths for our sample, and derive maps of the luminosity-weighted stellar age, metallicity and abundance ratio via stellar population models. From these data we find a wealth of structures either not seen or poorly resolved in the SAURON data, including a number of kinematically-decoupled cores (KDCs) in the centres of some galaxies. We compare the intrinsic size and luminosity-weighted stellar age of all the visible KDCs in the full SAURON sample, and find two types of components: kiloparsec-scale KDCs, which are older than $8 \mathrm{Gyr}$, and are found in galaxies with little net rotation; and compact KDCs, which have intrinsic diameters of less than a few hundred parsec, show a range of stellar ages from 0.5 - 15 Gyr (with 5/6 younger than 5 Gyr), are found exclusively in fast-rotating galaxies, and are close to counter-rotating around the same axis as their host. Of the 7 galaxies in the SAURON sample with integrated luminosity-weighted ages less than 5 Gyr, 5 show such compact KDCs, suggesting a link between counter-rotation and recent star-formation. We show that this may be partly due to a combination of small sample size at young ages, and an observational bias, since young KDCs are easier to detect than their older and/or co-rot ating counterparts.
\end{abstract}

Keywords. galaxies: elliptical and lenticular, cD; evolution; formation; kinematics and dynamics; stellar content; structure

$\dagger$ Poster available at: www.strw.leidenuniv.nl/ mcdermid/mcdermid_iau_2006_poster.pdf 